\title{
SELF-INCOMPATIBILITY IN RYEGRASS
}

\section{THE JOINT SEGREGATION OF S AND PGI-2 IN LOLIUM PERENNE L.}

\author{
M. A. CORNISH*f, M. D. HAYWARD + and M. J. LAWRENCE*
}

* Department of Genetics, University of Birmingham, Birmingham B15 2TT; †The Welsh Plant Breeding Station, Plas Gogerddan, Aberystwyth SY23 3EB

Received 1.viii.79

\section{SumMaRY}

An analysis of the data from six families of full-sibs shows that one of the incompatibility genes in perennial ryegrass is linked in its inheritance to the gene, $P G I-2$, which codes for the enzyme phosphoglucoisomerase. In the light of this evidence, we define $S$ as the gene that is linked to PGI-2. The best estimate of the frequency of recombination between $P G I-2$ and $S$ is $\hat{\mathrm{p}}=0 \cdot 1538 \pm 0 \cdot 0252$.

\section{INTRODUCTION}

Previous papers in this series have shown that self-incompatibility in Lolium perenne is determined by a pair of multi-allelic genes, $S$ and $Z$, which are inherited independently and whose effect in the pollen is gametophytic (Cornish, Hayward and Lawrence, 1979a, b). A number of genes coding for isozymes of different electrophoretic mobility within several enzyme systems have been identified recently in L. perenne (Hayward and McAdam, $1977 a, b$; Hayward and Balls, 1978), which raises the question of whether one or more of these genes are linked to $S$ or $Z$.

There are two reasons why the possibility of such linkages is of interest. Firstly, since the incompatibility genes are functionally identical, linkage of one to a convenient marker gene would enable $S$ to be distinguished from $Z$ without ambiguity. Secondly, were such a linkage to remain undetected, the inheritance of the marker gene could be open to serious misinterpretation, because of the special properties of incompatibility genes. Three of the genes which code for enzymes in ryegrass are regularly used to monitor both the segregation of the chromosomes in families of hybrid origin (Breese and Thomas, 1977) and the stability of populations during seed multiplication (Hayward and McAdam, loc. cit.), which underlines the practical implications of this point.

In the present paper, we consider the joint segregation of a gene, $P G I-2$, which codes for the enzyme phosphoglucoisomerase, with $S$ and $Z$.

\section{Materials AND MEthods}

Clonal duplicates of the plants of the first six families, $D$ and $E ; F$ and $G$; and $\mathrm{H}$ and $\mathrm{I}$, whose incompatibility phenotypes were reported by Cornish et al. (1979a), were, following the procedure of Hayward and McAdam (1977a), scored for their PGI-2 phenotype. One duplicate from family $\mathrm{H}$

$\ddagger$ Present address: School of Pharmacy and Pharmacology, University of Bath, Claverton Down, Bath BA2 7AY. 
and another from family $\mathrm{G}$ died before either could be scored. The parents of each of these families were also scored for their PGI-2 phenotype.

Hayward and McAdam (1977b) have shown that the PGI-2 polymorphism is controlled by a single gene, that the enzyme is dimeric and have found four allelic variants of the gene.

\section{Procedure}

We have shown in a previous paper (Cornish et al., 1979a) that families $\mathrm{D}$ and $\mathrm{E}$, and $\mathrm{H}$ and $\mathrm{I}$ arose from a cross of the type $S_{1.2} Z_{1.2} \times S_{3.4} Z_{3.4}$ and that the cross which gave rise to families $\mathrm{F}$ and $\mathrm{G}$ was of the type $S_{1 \cdot 2} Z_{1 \cdot 2} \times$ $S_{1 \cdot 3} Z_{3 \cdot 4}$. The results obtained by scoring the parents and their progeny for their PGI-2 phenotype showed that families D and E, and F and Gr were of the backcross type,

$$
\begin{aligned}
& {\left[\begin{array}{ll}
\text { Family D } & b c \times b b \\
\text { Family E } & b b \times b c
\end{array}\right.} \\
& {\left[\begin{array}{ll}
\text { Family F } & b b \times a c \\
\text { Family G } & a c \times b b
\end{array}\right.}
\end{aligned}
$$

and that the third cross was of the $\mathrm{F}_{2}$ type

$$
\left[\begin{array}{l}
\text { Family H } \\
\text { Family I }
\end{array} \quad a b \times a b\right.
$$

Taking these results together, therefore, families D, E, F and G are of the backcross type for the joint segregation of PGI-2 and $S$ or $Z$ and families $\mathrm{H}$ and I are incompletely classified intercrosses (Lawrence, Cornish and Hayward, 1979).

Now while the detection and estimation of linkage may be undertaken in the usual way in the backcross families, we are faced with the same problem here as that encountered in the previous paper (Cornish et al., $1979 b$ ), namely that we have no direct knowledge of the incompatibility genotypes of the parents. Thus the cross that gave rise to family $\mathbf{F}$, for example, could have been either $b S_{1} / b S_{2} \times a S_{1} / c S_{3}$ or $b S_{1} / b S_{3} \times a S_{1} / c S_{2}$ and similarly for the $Z$ locus. For this reason, we have to make two independent tests for linkage for each incompatibility gene. If linkage is present, only one of each pair of $\chi^{2}$ 's is expected to be significant, because the other concerns the joint segregation of $P G I-2$ and $S$ (or $Z$ ) alleles that have come from different sides of the cross and cannot, therefore, be linked in their inheritance. However, as in the previous paper (Cornish, loc. cit.), because we shall be carrying out two tests for linkage (for either incompatibility gene), the probability of an error of the first kind is approximately twice the correponding probability for a single test (the exact probability is 1.9 times the latter). Hence in testing the null hypothesis of no linkage between PGI-2 and $S$ (or Z) it is appropriate to choose $\mathrm{P}=0.025$ and $\mathrm{P}=0.005$ (strictly, $\mathrm{P}=0.0263$ and $\mathrm{P}=0.0053$ ) as the levels of significance rather than the conventional 5 per cent and 1 per cent levels.

In the case of families $\mathrm{H}$ and $\mathrm{I}$, it is possible to test for and to obtain estimates of both male and female linkage, because $P G I-2$, as well as $S$ (or Z), has segregated on each side of the cross. The expected composition of a 
TABLE 1

The expected composition of the progeny of a cross between an $\mathrm{aS}_{1} / \mathrm{bS}_{2}$ female and an $\mathrm{as}_{3} / \mathrm{bS}_{4}$ male. All frequencies in the table should be divided by 4

\begin{tabular}{|c|c|c|c|}
\hline$S_{1} S_{3}$ & $S_{1} S_{4}$ & $S_{2} S_{3}$ & $S_{2} S_{4}$ \\
\hline $\begin{array}{c}a a S_{1} S_{3} \\
\mathrm{qfq}_{\mathrm{m}} \\
\mathrm{n}_{11}\end{array}$ & $\begin{array}{c}a a S_{1} S_{4} \\
\mathrm{q}_{\mathrm{pm}} \\
\mathrm{n}_{12}\end{array}$ & $\begin{array}{c}a a S_{2} S_{3} \\
\mathrm{p}_{\mathrm{mq}} \\
\mathrm{n}_{13}\end{array}$ & $\underset{\substack{a t p_{m} \\
\mathrm{n}_{14}}}{a S_{2} S_{4}}$ \\
\hline $\begin{array}{c}a b S_{1} S_{3} \\
\mathrm{p}_{\mathrm{p}} \mathrm{q}_{\mathrm{m}}+\mathrm{q}_{\mathrm{q}} \mathrm{p}_{\mathrm{m}} \\
\mathrm{n}_{21}\end{array}$ & $\begin{array}{c}a b S_{1} S_{4} \\
\mathrm{p}_{t} \mathrm{p}_{\mathrm{m}}+\mathrm{q}_{\ell \mathrm{q}} \mathrm{q}_{\mathrm{m}} \\
\mathrm{n}_{22}\end{array}$ & $\begin{array}{c}a b S_{2} S_{3} \\
\mathrm{p}_{\imath \mathrm{p}}+\mathrm{q}_{\ell} \mathrm{q}_{\mathrm{m}} \\
\mathrm{n}_{2 \mathrm{~s}}\end{array}$ & $\begin{array}{c}a b S_{2} S_{4} \\
\mathrm{p}_{t} \mathrm{q}_{\mathrm{m}}+\mathrm{q}_{\mathfrak{t} \mathrm{p}_{\mathrm{m}}} \\
\mathrm{n}_{24}\end{array}$ \\
\hline $\begin{array}{c}b b S_{1} S_{3} \\
\mathrm{p}_{2 p_{12}} \\
\mathrm{n}_{31}\end{array}$ & $\begin{array}{c}b b S_{1} S_{4} \\
\mathrm{p}_{\mathrm{q}} \mathrm{q}_{\mathrm{m}} \\
\mathbf{n}_{\mathbf{3} 2}\end{array}$ & $\begin{array}{c}b b S_{2} S_{3} \\
\mathrm{q}_{t p} \\
\mathrm{n}_{33}\end{array}$ & $\begin{array}{c}b b S_{2} S_{4} \\
\mathrm{q}_{\mathrm{pq}} \\
\mathrm{n}_{34}\end{array}$ \\
\hline
\end{tabular}

family of this type is shown in table 1. Four tests of linkage can be made in respect of $P G I-2$ and each of the incompatibility genes as follows:

$\begin{array}{ll}\text { Female linkage } & \chi^{2}(1)=[2(C+D-E-F)]^{2} / 2 \mathrm{n} \\ \text { Male linkage } & \chi^{2}(1)=[2(C-D+E-F)]^{2} / 2 \mathrm{n} \\ \text { Joint }(ð+\text { ) linkage } & \chi^{2}(1)=[4(C-F)]^{2} / 4 \mathrm{n} \\ \text { Heterogeneity }(ð-\text { o }) \text { linkage } & \chi^{2}(1)=[4(D-E)]^{2} / 4 \mathrm{n}\end{array}$

where $C=\mathrm{n}_{11}+\mathrm{n}_{34} ; D=\mathrm{n}_{21}+\mathrm{n}_{33} ; E=\mathrm{n}_{23}+\mathrm{n}_{32} ; F=\mathrm{n}_{14}+\mathrm{n}_{31} ;$ and $\mathrm{n}=$ number of plants in the family (Lawrence et al., 1979). In practice, the last two items in this list of $\chi^{2}$ s are likely to be of greatest interest. Indeed, there is little point in obtaining the female and male linkage $\chi^{2}$ 's in present circumstances, since the parents of families $H$ and $I$ have been used both as female and male. In addition to these tests of linkage, tests of the segregation of $a a: a b: b b$ and of $S_{1}: S_{2}$ and $S_{3}: S_{4}$ may be carried out on the row and column totals of the table respectively.

Because of our ignorance about the incompatibility genotype of the parents it is necessary to consider four different crosses, any of which could have given rise to these families. For the $S$ locus, these crosses are:

$$
\begin{aligned}
& a S_{1} / b S_{2} \times a S_{3} / b S_{4} \\
& a S_{1} / b S_{2} \times a S_{4} / b S_{3} \\
& a S_{2} / b S_{1} \times a S_{3} / b S_{4} \\
& a S_{2} / b S_{1} \times a S_{4} / b S_{3}
\end{aligned}
$$

For similar reasons to those given in the previous paper (Cornish et al., $1979 b)$, the detection of linkage is not affected by the arrangement of the genes in the parents, except that the distinction between the joint and heterogeneity $\chi^{2}$ 's becomes arbitrary, since these items are interchangeable (the same is true of the female and male items). If linkage is present in the data, either the joint or the heterogeneity $\chi^{2}$ (or possibly both) is expected to be significant. Once linkage has been detected, it should be possible by inspecting the data to deduce which of the four types of cross has given rise to the family in question and hence to decide which $\chi^{2}$ of the pair is the joint and which is the heterogeneity item. Once again the appropriate levels of significance for tests of linkage on the data of an incompletely classified intercross where the genotypes of the parents are not known completely, are 0.025 and 0.005 respectively.

The methods used for testing the homogeneity of linkage when $p \neq 0.5$ 
and for combining estimates of linkage are those given by Mather (1951) and used by Lawrence et al. (1979).

\section{Results}

(i) Families $F$ and $G$

The data and analyses of these two families are shown in table 2. Only one of the five single factor ratios departs significantly from the expected $1: 1$ ratio $\left(Z_{1}: Z_{2}, \chi_{(1)}^{2}=22.730, P<0.001\right)$ so that the tests for linkage will not be disturbed by differential viability.

\section{TABLE 2}

(a) The data from families $F$ and $G$ arranged so as to allow tests of linkage between the PGI-2 alleles, a and $\mathrm{c}$, with each of the four pairs of incompatibility alleles in turn. (b) Linkage $\chi^{2}$ 's for each of these four comparisons in each of the two families ( $, P=0.025-0.005, * * *, P<0.001)$

(a) $a: c$

\begin{tabular}{lccccc}
$a: c$ & & & & & \\
with & Family & $a S_{1}$ & $c S_{1}$ & $a S_{2}$ & $c S_{2}$ \\
$S_{1}: S_{\mathbf{2}}$ & $\mathrm{F}$ & 20 & 3 & 2 & 12 \\
& $\mathrm{G}$ & 10 & 6 & 2 & 11 \\
\cline { 3 - 6 } & & $a S_{1}$ & $c S_{1}$ & $a S_{3}$ & $c S_{3}$
\end{tabular}

$\begin{array}{rrrrrr}S_{1}: S_{3} & \text { F } & 8 & 11 & 14 & 4 \\ & \text { G } & 5 & 5 & 7 & 12\end{array}$

\begin{tabular}{cccccc} 
& & $a Z_{1}$ & $c Z_{1}$ & $a Z_{2}$ & $c Z_{2}$ \\
$Z_{1}: Z_{2}$ & $\mathrm{~F}$ & 19 & 14 & 3 & 1 \\
& $\mathrm{G}$ & 6 & 8 & 6 & 9 \\
\hline & & $a Z_{3}$ & $c Z_{3}$ & $a Z_{4}$ & $c Z_{4}$
\end{tabular}

$\begin{array}{rrrrrr}Z_{3}: Z_{4} & \mathrm{~F} & 14 & 5 & 8 & 10 \\ & \mathrm{G} & 5 & 10 & 7 & 7\end{array}$

(b)

\begin{tabular}{ccc}
$a: c$ & \multicolumn{2}{c}{ Family } \\
with & $\overbrace{\mathrm{F}}$ & $\mathrm{G}$ \\
$S_{1}: S_{2}$ & $19 \cdot 703 * * *$ & $5.828 *$ \\
$S_{1}: S_{3}$ & 4.567 & 0.862 \\
$Z_{1}: Z_{2}$ & 0.243 & 0.034 \\
$Z_{3}: Z_{4}$ & 3.270 & 0.862
\end{tabular}

N.B. $\mathrm{P}\left(\chi^{2}(1) \geqq 5.024\right)=0.025$ and $\mathrm{P}\left(\chi^{2}(1) \geqq 7 \cdot 789\right)=0.005$.

There is no evidence of linkage between $P G I-2$ and the incompatibility gene labelled $Z$ in these families, since none of the four $\chi^{2}$ 's in question are significant. Both of the $\chi^{2}$ 's testing for linkage between PGI-2 and the $S_{1}, S_{2}$ pair of alleles are significant. Furthermore, the intensity of linkage appears to be the same in each parent (heterogeneity of reciprocals $\chi^{2}{ }_{(1)}=$ $1 \cdot 289, \mathrm{P}=0 \cdot 30-0 \cdot 20)$. We conclude, therefore, that PGI-2 is linked to the gene labelled $S$ and that the cross that gave rise to family $F$ was $b S_{1} / b S_{3} \times a S_{1} / c S_{2}$. The joint estimate of linkage obtained by pooling the data over reciprocal families is $\hat{p}=0 \cdot 1970 \pm 0 \cdot 0490$. 
(ii) Families $D$ and $E$

The occurrence of self-pollinated offspring in family $\mathrm{D}$ enabled us to deduce that the $S_{1}, S_{2}$ pair of incompatibility alleles must have descended from the female side of this family and hence from the male side of family $\mathrm{E}$ (Cornish et al., 1979a). Since this parent is also heterozygous for the PGI-2 alleles, $b$ and $c$, there is no need to test for linkage between these and the alternative allelic pair, $S_{3}, S_{4}$, in these families.

The data and analyses of these families are shown in table 3 . Tests of the single factor ratios show that while both $S_{1}: S_{2}$ and $b: c$ are disturbed in

\section{TABLE 3}

(a) The data from families $D$ and $E$ arranged so as to allow tests of linkage between the PGI-2 alleles, $\mathrm{b}$ and $\mathrm{c}$, with each of three pairs of incompatibility alleles in turn. (b) Linkage $\chi^{2}$ 's for each of these three comparisons in each of the two families. (The test for linkage between PGI-2 and $S_{1}$, $S_{2}$ in family $D$ is a corrected $2 \times 2$ contingency $\chi^{2}$ )

(a) $b:$

\begin{tabular}{lcrrrr}
$b: c$ & & & & \\
with & Family & $b S_{1}$ & $c S_{1}$ & $b S_{2}$ & $c S_{2}$ \\
$S_{1}: S_{2}$ & $\mathrm{D}$ & 5 & 3 & 1 & 20 \\
& $\mathrm{E}$ & 14 & 6 & 2 & 12 \\
\cline { 3 - 6 }$Z_{1}: Z_{2}$ & $\mathrm{D}$ & 3 & 7 & 3 & 16 \\
& $\mathrm{E}$ & 8 & 13 & 8 & 5 \\
& & $b Z_{1}$ & $c Z_{1}$ & $b Z_{2}$ & $c Z_{2}$ \\
& & & $c Z_{3}$ & $b Z_{4}$ & $c Z_{4}$ \\
$Z_{3}: Z_{4}$ & $\mathrm{D}$ & 2 & 8 & 4 & 15 \\
& $\mathrm{E}$ & 8 & 7 & 8 & 11 \\
\hline & & & & &
\end{tabular}

(b)

\begin{tabular}{|c|c|c|}
\hline \multirow{2}{*}{$\begin{array}{l}b: c \\
\text { with }\end{array}$} & \multicolumn{2}{|c|}{ Family } \\
\hline & D & $\mathbf{E}$ \\
\hline $\begin{array}{l}S_{1}: S_{2} \\
Z_{1}: Z_{2} \\
Z_{3}: Z_{4}\end{array}$ & $\begin{array}{l}(8.514 * *) \\
2.793 \\
0.862\end{array}$ & $\begin{array}{l}9 \cdot 529 * * \\
1.882 \\
0.471\end{array}$ \\
\hline
\end{tabular}

family $\mathrm{D}\left(\chi^{2}{ }_{(1)}=5.828, \mathrm{P}=0.02-0.01\right.$ and $\chi^{2}{ }_{(1)}=9.966, \mathrm{P}=0.01-0.001$ respectively), neither of these ratios departs significantly from $1: 1$ in the reciprocal family $\mathrm{E}$. Because we are not able to account for this inconsistency, the data from family $\mathbf{E}$ must be regarded as the more trustworthy. We note that the only significant $\chi^{2}$ in this family concerns the joint segregation of $P G I-2$ with the $S_{1}, S_{2}$ pair of alleles, so that in this family also, $P G I-2$ appears to be linked to the gene labelled $S$. The cross that gave rise to family $\mathrm{E}$ was thus $b S_{3} / b S_{4} \times b S_{1} / c S_{2}$ and the estimate of linkage is $\hat{p}=0.2353 \pm 0.0727$.

Lastly, though we may have doubts about the reliability of the data from $\mathrm{D}$, the evidence from this family is at least consistent with that from family $\mathrm{E}$ in indicating linkage between the same pairs of alleles.

\section{(iii) Families $H$ and $I$}

The results obtained from these families, arranged in a similar way to table 1 , are shown in tables 4 and 5 . None of the single factor ratios depart 
significantly from the expected $1: 1$ in the case of the $S$ and $Z$ alleles or from the expected $1: 2: 1$ in the case of the PGI-2 genotypes, $a a: a b: b b$.

\section{TABLE 4}

The data of family $H$ arranged in a similar way to table 1. Entries for $\mathrm{S}$ are shown in the upper half of each cell in the table and those for $\mathrm{Z}$ in the lower half. The heterogeneity $\chi^{2}$ for PGI-2 and $\mathrm{S}$ has been calculated about the joint estimate of linkage, $\hat{p}=0.1238$

$\left.\begin{array}{cccccc} & S_{1} S_{4} & S_{1} S_{3} & S_{2} S_{4} & S_{2} S_{3} & \text { Row } \\ \multirow{5}{*}{a a} & Z_{1} Z_{3} & Z_{1} Z_{4} & Z_{2} Z_{3} & Z_{2} Z_{4} & \text { Totals } \\ & 6 & 1 & 0 & 0 \\ a b & 4 & 2 & 1 & 0 \\ & 2 & 5 & 6 & 2 \\ b b & 5 & 5 & 3 & 2 \\ & 0 & 2 & 0 & 6 \\ \text { Column } & 4 & 0 & 2 & 2 \\ \text { Totals } & 8 & 8 & 6 & 8 \\ & 13 & 7 & 6 & 4\end{array}\right\}$

(a) PGI-2 and $S$

Joint $\chi^{2}(1) \quad=19 \cdot 200, \mathrm{P}<0 \cdot 001$

Heterogeneity $\chi_{(1)}^{2}=0.307, \mathrm{P}=0.7-0.5$

(b) $P G I-2$ and $Z$

Joint $\chi^{2}(1) \quad=0.533, P=0.5-0.3$

Heterogeneity $\chi^{2}(1)=1 \cdot 200, \mathrm{P}=0 \cdot 3-0 \cdot 2$

TABLE 5

The data of family I arranged in a similar way to table 1 . Other details as for table 4

$\left.\begin{array}{cccccc} & S_{1} S_{4} & S_{1} S_{3} & S_{2} S_{4} & S_{2} S_{3} & \text { Row } \\ & Z_{1} Z_{3} & Z_{2} Z_{3} & Z_{2} Z_{4} & Z Z & \text { Totals } \\ a a & 7 & 0 & 1 & 1 \\ & 6 & 1 & 2 & 0 \\ a b & 1 & 10 & 9 & 0 \\ & 4 & 3 & 7 & 6 \\ b b & 1 & 0 & 1 & 6 \\ & 3 & 0 & 1 & 4 \\ \text { Column } & 9 & 10 & 11 & 7 \\ \text { Totals } & 13 & 4 & 10 & 10\end{array}\right\}$

(a) $P G I-2$ and $S$

Joint $\chi^{2}(1) \quad=13.081, \mathrm{P}<0.001$

Heterogeneity $\chi^{2}(1)=0$

(b) $P G I-2$ and $Z$

$\begin{array}{ll}\text { Joint } \chi^{2}(1) & 5 \cdot 297, \mathrm{P}=0 \cdot 025-0 \cdot 020 \\ \text { Heterogeneity } \chi^{2}(1)= & 0\end{array}$

In both families $\mathrm{H}$ and $\mathrm{I}$ there is unambiguous evidence of linkage between PGI-2 and the incompatibility gene labelled $S$. Furthermore, the intensity of linkage is consistent over sexes and reciprocal families $\left(\chi^{2}{ }_{1}\right)=$ $0 \cdot 150, \mathrm{P}=0.7-0.5$, calculated about the joint estimate of linkage, for the latter). The cross giving rise to family $\mathrm{H}$ was $a S_{1} / b S_{2} \times a S_{4} / b S_{3}$ and the joint estimate of linkage obtained by pooling the data over sexes and families is $\hat{p}=0.1114 \pm 0.0289$. 
The evidence concerning the joint segregation of $P G I-2$ and $Z$ is equivocal. On the one hand, while there is no evidence of linkage in family $\mathrm{H}$, the joint $\chi^{2}{ }_{(1)}$ in I is just significant. At first sight we might wish to attribute this outcome to chance alone, particularly since we have not found evidence of linkage between these genes in any other family. On the other hand, the joint test of linkage calculated on the data obtained by pooling over families is also just significant $\left(\chi^{2}{ }_{(1)}=4.836, \mathrm{P}=0.05-0.02\right.$, the conventional levels of significance applying in this case) and linkage appears to be consistent over both sexes and families $\left(\chi^{2}{ }_{(1)}=0.973, P=0.5-0.3\right)$. Furthermore, it may not be a coincidence that we have detected linkage in these families both because they are incompletely classified intercrosses, which are more efficient than backcrosses for the detection and estimation of linkage (Lawrence et al., 1979) and because on the evidence of the joint segregation of $P G I-2$ and $S$, linkage may be tighter in this cross than in other families. On this argument, the cross that gave rise to family $\mathrm{H}$ was $a Z_{1} / b Z_{2} \times a Z_{3} / b Z_{4}$ and the joint estimate of linkage obtained by pooling the data over sexes and families is $\hat{p}=0 \cdot 3645 \pm 0.0552$. Though, on the present evidence, this conclusion must be regarded as very tentative, it does suggest that $S$ and $Z$ may be carried on the same chromosome, albeit at a distance apart which is great enough to ensure that they assort independently.

\section{(iv) The homogeneity of linkage over crosses}

We have shown that linkage between PGI-2 and $S$ is homogeneous over reciprocals in families $F$ and $G$ and in families $H$ and $I$ and, in the case of the latter pair, over sexes also. To complete this analysis, we need to combine the three estimates

and

$$
\begin{aligned}
& \hat{p}_{\mathrm{FG}}=0.1970 \pm 0.0490 \\
& \hat{p}_{\mathrm{E}}=0.2353 \pm 0.0727
\end{aligned}
$$

$$
\hat{p}_{\mathrm{HI}}=0 \cdot 1114 \pm 0.0289
$$

to obtain an overall joint estimate of linkage between PGI-2 and $S$, and to find out whether linkage is homogeneous over crosses about this joint estimate. The overall estimate of linkage obtained by pooling the data over families is $\hat{p}=0.1583 \pm 0.0252$ and the heterogeneity $\chi^{2}$ is not significant $\left(\chi^{2}{ }_{(2)}=4 \cdot 076, \mathrm{p}=0 \cdot 2-0 \cdot 1\right)$.

\section{Discussion}

Since the incompatibility genes $S$ and $Z$ are functionally indistinguishable it is not possible to recognise either without ambiguity, on incompatibility evidence alone. We have, however, obtained unambiguous evidence from each of the three crosses examined that PGI-2 is linked to the gene which we had previously called $S$ and we can now, therefore, define $S$ as the incompatibility gene that is linked in its inheritance to PGI-2.

This is, of course, not the first time that linkage has been found between an incompatibility gene and another; de Nettancourt (1977) gives seven such cases in homomorphic systems of self-incompatibility. It is, however, the first case of linkage involving a gene of a two-locus system of self-incompatibility and, indeed, the first case also of linkage to an isozyme locus. 
Furthermore, since $P G I-2$ is now known to be carried on chromosome 6 in L. perenne (E. J. Lewis, personal communication), it follows that $S$ must be carried on this chromosome also. The evidence of families $\mathrm{H}$ and $\mathrm{I}$ indicates that $Z$ may also be located on this chromosome.

Lastly, the ease with which it has been possible to detect linkage between $S$ and $P G I-2$ removes once and for all any doubts that might remain about the accuracy of the classification of the incompatibility phenotypes of the plants in these families and hence confirms the conclusion that $L$. perenne has a two-locus system of self-incompatibility.

Acknowledgments.-We are again greatly indebted to Dr J. S. Gale and Professor Sir Kenneth Mather for much useful comment on the problems of the detection and estimation of linkage. We also wish to acknowledge the receipt of a Science Research Council C.A.S.E. award which enabled the work described in this paper to be carried out.

\section{REFERENCES}

BREeSE, E. L., AND THOMAS, A. C. 1977. Monitoring chromosome segregation in Lolium multiflorum $\times$ Lolium perenne amphiphloids. Rep. Welsh Pl. Breed. Stn. for 1976, 29-32.

CORNISH, M. A., HAYWARD, M. D., AND LAWRENCE, M. J. 1979a. Self-incompatibility in ryegrass. I. Genetic control in diploid Lolium perenne L. Heredity (in the press).

CORNISH, M. A., HAYWARD, M. D., AND LAWRENCE, M. J. 1979b. Self-incompatibility in ryegrass. II. The joint segregation of $S$ and $Z$ in Lolium perenne L. Heredity (in the press).

HAYWARD, M. D., AND BALLS, T. 1978. Isoenzyme polymorphism in natural populations of Lolium perenne. Rep. Welsh Pl. Breed. Stn. for 1977, 43-45.

HAYWARD, M. D., AND MCADAM, N. J. 1977a. Isozyme polymorphism as a measure of distinctiveness and stability in cultivars of Lolium perenne. Z. Pfanzenzuchtg., 79, 59-68.

HAYWARD, M. D., AND MCADAM, N. J. 1977b. Genetic control of isoenzyme phenotypes in L. perenne. Rep. Welsh Pl. Breed. Stn. for 1976, 28-29.

LAWRENCE, M. J., CORNiSH, M. A., AND HAYWARD, M. D. 1979. Simultaneous estimation of male and female linkage from a single intercross family. Heredity (in the press).

Mather, K. 1951. The Measurement of Linkage in Heredity. Methuen, London. De nettancourt, D. 1977. Incompatibility in Angiosperms. Springer-Verlag, Berlin. 